\title{
Comparative Analysis of Techniques for the Recognition of Stabbed Wound and Accidental Wound Patterns
}

\author{
Dayanand G. Savakar \\ Department of Computer Science \\ Rani Channamma University, Belagavi \\ INDIA
}

\author{
Anil Kannur \\ Department of Computer Engineering \\ A G Patil Institute of Technology, Solapur \\ INDIA
}

\begin{abstract}
The Paper proposed a comparative analysis of wound patterns for the process of recognition whether the wound is stabbed wounds using any sharp metals or accidental wounds. The analysis is on the basis of characteristics of wounds in terms of parameters like shape, size and crime scene. And this paper also presents analysis of different segmentation techniques, possible better combination of features to extract for the recognition and finally analysis on different recognition methodologies. Different schemas of recognition are presented in which combination of different segmentation algorithms, features vectors and two approaches of classifiers, and also the comparative analysis of these schemas is discussed. Based on comparative analysis, the combination of three stage techniques of recognition has given results in diverse. From these schemas of recognition, the structural method has given better results compared to the other schemas on the available database of 500 images of pattern consisting of stabbed wounds and accidental wounds. The authenticated experiments out-turns the superiority of the proposed approach over the other approach considered in this work and also compares and suggest the false positive recognition verses false negative recognition. The proposed methodology has given better results compared to traditional method and will be helpful in forensic and crime investigation.
\end{abstract}

\section{General Terms}

Pattern Recognition, Image Processing, Computational Forensic Science.

\section{Keywords}

Classifiers, Features, Patterns, Segmentation, Selection, Wounds.

\section{INTRODUCTION}

The Combination of Computer Science and Forensic Science has evolved in recent years, which has given new dimension to the investigation known as Computational Forensic Science. Computational forensics science is pertaining to evidence found in crime scene, computers and digital storage media. The goal is to examine digital media and evidences with the purpose of recognizing, conserving, analyzing and presenting actualities and views about the digital information and evidences. In addition to this, Computational forensic science also includes the analysis of biometric, handwriting and patterns of shoes and tires. In recent years, one more area added to the computational forensic science is bitemark analysis which is field of forensic dentistry. This paper proposes to add one more feather to the computational forensic science, i.e., recognition of weapons based on the wound patterns (images) which are collected from the victims' body in most of homicidal cases. The survey provides adequate descriptions of wounds on victims, including defensive wounds, caused by knives and other sharp metals. In several instances, these wounds and the physical evidence have proven of great significance in the resolution of the case. Many of the forensic science papers defined wound patterns were written by doctors; they generally see only the wounds of the victim, and not the surviving attacker. If anyone documents or acquires the wounds, undoubtedly it will be the police or forensic personnel. Careful examination of a body frequently reveals the presence of well-known patterned wounds that prove to be extremely important in the reconstruction of a crime or accident. The research purpose is to demonstrate the critical studies of wound patterns in a brutal murder led to the recognition of the murder weapon.

Image processing and pattern classification plays an important role in this proposed problem that can be added to computational forensic science. Image processing comprises steps from image acquisition from various devices to image understanding. Image understanding involves automated system of extracting data from wound patterns in an image. As main target of image processing, automatic image understanding is a vital step of the image processing. In the process of image understanding, the automatic localization and recognition of wound patterns in an image is still a great challenge. In the projected application, the extracted data will play important in the performances of the methodology. This is required that localization must be precise and with accurate recognition. Many methodologies are proposed by researchers to accomplish this recognition, but still it is problematic effort to equate the performance of the methodologies. For the input image given, whether a segment identified by methodologies are accurate or not. If the input images used for these methodologies application to common intelligence, better ratio of height/width and the size of the perceived bounding box and none of them places the same parameters forward. Besides, many methodologies are proposed for various purposes, such as segmentation algorithms to image retrieval/recognition algorithms, can be found in the literature and should reveal themselves relevant for better performances. How can one choose a better sequence of image processing and pattern classification chain working on individual results to consistently evaluate methodologies? The target of proposed work is to show analysis that makes comparison of segmentation techniques, feature selection and recognition or classification algorithms. The queries that may raise for the work includes: (i) Which segmentation technique will give better region-of-interest? (ii) Which is the best combination of features to select? (iii) Which recognition or classification algorithm will be better to get higher accuracy? It has been considered in this work, a comparative analysis of the techniques for the recognition of stabbed wounds and accidental wounds patterns with weapons of cause. 


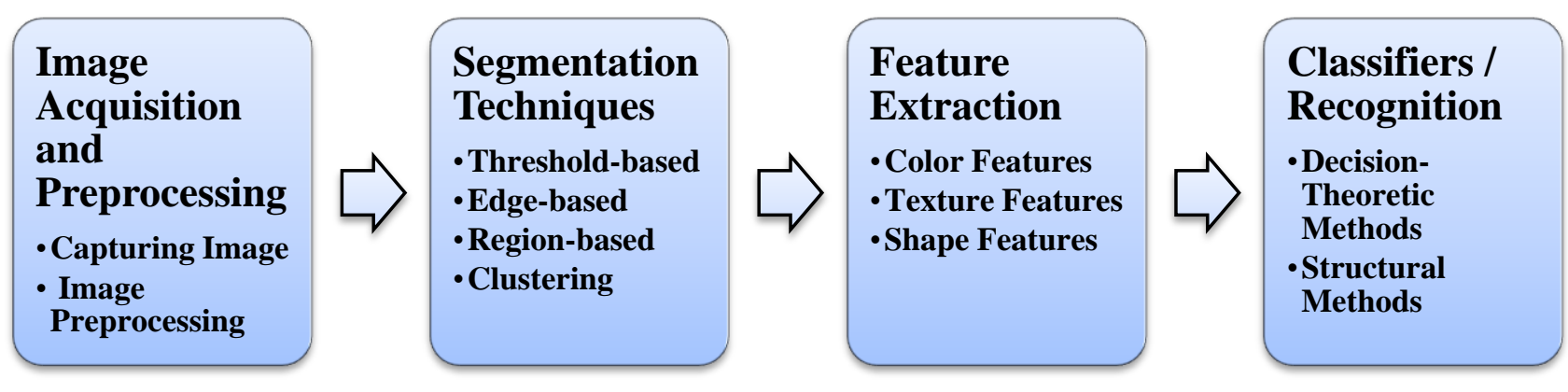

Fig 1: Flowchart of different Schemas of recognition

The process of evaluating the techniques and algorithms, exploits the only input data used by the image interpretation algorithm, i.e., the original image, and gives a score of coherence that quantifies the possibility that the result given by the algorithm is correct. By the way, this evaluation gives an adequacy score between results and the corresponding ground truths. This score is of course more reliable but requires the definition of a ground truth for each input image. The evaluation of an algorithm can be either the evaluation of a single result or a global evaluation. In the case where there is need to evaluate the global performance of an algorithm, the objective is to study its general behavior considering different parameters, and to know what kind of alteration it is able to face to illumination changes, presence of occlusion, etc. The evaluation process permits us to study the influence of internal parameters of the algorithm and to optimize them. The goal is then to compute for each single image some performance measures on different results obtained by changing the parameters value. To realize a reliable evaluation, there is need of an evaluation database with enough images to be representative of most cases that the algorithm is used for. To study the performances of techniques and algorithms, most of the organized competitions work in a supervised context and provide ground truth annotations across all the proposed databases. Finally, conclusions and perspectives of this study are given.

\section{LITERATURE SURVEY}

Song Bo (2012) has proposed a system that can automatically and accurately identify the region of a chronic wound and is capable of automatic image segmentation and wound region identification (using ANN, MLP and RBF). Several commonly used segmentation methods are utilized with their parameters fine-tuned automatically to obtain a collection of candidates wound regions. Hugar B.S. et al., (2012) have proposed work to determine specific patterns and distribution of defense injuries, this study was conducted on 121 homicidal deaths which showed defense injuries in 40 cases. The Image comparison technique is used in identifying the patterns on the wounds. Gitto L., et. al., (2012) presented a peculiar case of homicide committed with a sharp instrument. The techniques used is autopsy investigation, an unusual damage was found in the vicinity of the fatal wound to the neck, suggesting that the wound was inflicted with a great force, using a sharp object with a peculiar shape. A comparative study between the two weapons was performed in order to determine the compatibility with the abovementioned damage. Ying Bai and Dali Wang (2011) proposed this research to develop a universal model and system to effectively assess, evaluate and identify the optimal weapons from a large collection of available weapon systems that have multiple criteria based on a fuzzy multiple criteria decision making FMCDM model. Kaliszan M. et. al., (2011) presented an experiment with a knife seized as material evidence in a homicide case. A comparison of the morphological features of the stab wound of the abdomen found during autopsy and the morphological features of the wound of the abdomen resulting from the above experiment showed a similarity between their shapes and the location and size of the linear skin abrasions in the edges of both wounds. Francisco Veredas et. al., (2010) have proposed a hybrid approach based on neural networks and Bayesian classifiers, that is used in the design of a computational system for automatic tissue identification in wound images. A mean shift procedure and a region-growing strategy are implemented for effective region segmentation. Color and texture features are extracted from these segmented regions. Finally, a Bayesian committee machine is formed by training a Bayesian classifier to combine the classifications of the neural networks. Specific heuristics based on the wound topology are designed to significantly improve the results of the classification. $\mathrm{Li}$ Dongguang, (2008) worked on the characteristic markings on the cartridge case and projectile of a fired bullet are created when it is fired. By analyzing features within such a set of firearm fingerprints, it will be possible to identify not only the type and model of a firearm, but also every individual weapon as effectively as human fingerprint identification. A new analytic system based on fast Fourier transform (FFT) for identifying the projectile specimens by the line-scan imaging technique is proposed in this paper. Pretty I.A., and Turnbull M.D., (2001) described the dental uniqueness between two bite mark suspects by using overlays were produced using a modified method, despite the similarities noted above, both of the overlays demonstrated unique features, and it was not until the overlays were applied to the bite mark that the similarities became apparent. Both forensic dentists carried out metric and overlay analyses of the suspects. Quatrehomme G., (1999) described wound in terms of its shape, beveling, and location. Dimensions of both entrance and exit wounds were measured to the nearest millimeter. Surface area was calculated using the necessary formula for its geometric shape. An exit/entry surface area index was computed from these surface areas. The trajectory of the bullet was estimated when possible and the caliber of the bullet was noted when available. The path of the projectile was determined by noting its general direction. When there was no exit, direction was estimated from the wound shape and autopsy report. West M. et. al., (1992) noted that the absorption of electromagnetic energy or light by melanin pigment gradually increased from the red end of the spectrum as the violet end was reached. The absorption continued to increase even into the ultraviolet range. He reported that melanin and its breakdown products actually masked other materials that would have absorbed both visible and ultraviolet radiation. 


\section{CHARACTERISTICS OF WOUNDS}

There are many reasons for the wounds but in this work, considered two types of wounds for the study: stabbed wounds and accidental wounds. These wounds are created when any metal is used to attack or accidental impact of the metals (or any other) on human. These wounds relate to the shape of weapon used or any metal, that definitely leaves behind the characteristics which help in the recognition process. Stab wound is produced when force is delivered along the long axis of a narrow or pointed object such as knife, dagger, chisel, sword, sickle, etc. The characteristics of the wounds are:

- Margins of the wounds are created based on edges of weapons that are clean and inverted. The margins can be everted if wound is produced on fatty area such as abdomen and gluteal region. Usually there is no abrasion or bruise of the margins, but in full penetration abrasion and bruise may be found and may be abraded and ragged if the cutting edge is blunt.

- Length is slightly less than the width of the weapon upto which it has been driven in, because of stretching of the skin. And the maximum possible width of the knife can be approximately determined if the edges of a gapping wounds are brought together.

- Depth is greater than the width and length of the external injury. The depth is usually equal to or less than the length of the blade that was used in producing it, but on yielding surfaces like the anterior wall, the depth of the wound may be greater, because the force of thrust may press the tissues underneath. Depth of stab wound depends on condition of the knife's sharpness of tip, thin slender, double edged knife, resistance offered by the organs or tissues, clothing on human body, speed of the thrust of the knife and stretched skin or lax skin.

- Shape of the wounds depends on the type of implement, cutting surface, sharpness, width and shape of the weapon. It also depends on place or region of body (condition of the tension or relaxation of the skin) stabbed by person in movement or not and also the depth of insertion and withdrawal.

Overall opinion of the stabbed wounds with respect to the external and internal appearance of a stab wound, that helps us to give an opinion upon: the dimensions of the weapon, type of the weapon, the taper of the blade, movement of the blade in the wound, the depth of the wound, the direction of the stab and amount of the force is determined by the depth of insertion. Weapon Examination is carried in terms of the length, width and thickness of the blade. Whether it is single edged or double edged, degree of taper from hilt to tip and sharpness of the extreme tip of the blade and cutting edge. The Legal importance of the examination describes the shape of the wound may indicate the class and type of weapon, the depth of the wound will indicate the force of penetration and dimension and direction of wound indicate the relative positions of the assailant and victim.

\subsection{Comparison of Stabbed wounds with Accidental wounds}

Position, number and direction of the wound may indicate manner of production i.e. homicidal (stabbed) or accidental. Most stab wounds are homicidal, especially found in an in accessible area and accidental wounds are rare. The following factors are helpful in determining whether the wound is homicidal or accidental (Accidental, self-inflicted or inflicted by others): The nature, direction, extent and situation of the wound, the presence of the foreign matter in the wound, the nature of the suspected weapon and the scene of the crime.

Table 1. Comparison of Stabbed wounds (Homicidal) and Accidental Wounds

\begin{tabular}{|c|c|c|}
\hline $\begin{array}{l}\text { Characters or } \\
\text { Properties }\end{array}$ & $\begin{array}{l}\text { Stabbed Wounds } \\
\text { or Homicidal } \\
\text { Wounds }\end{array}$ & $\begin{array}{l}\text { Accidental } \\
\text { Wounds }\end{array}$ \\
\hline $\begin{array}{l}\text { Number of } \\
\text { wounds }\end{array}$ & $\begin{array}{l}\text { Frequently } \\
\text { Multiple, may be } \\
\text { Single }\end{array}$ & $\begin{array}{l}\text { Usually Single, } \\
\text { May be Multiple }\end{array}$ \\
\hline Site or Body Part & May be Anywhere & May be Anywhere \\
\hline $\begin{array}{l}\text { Tentative } \\
\text { Wounds }\end{array}$ & $\begin{array}{l}\text { May be present } \\
\text { rarely but away } \\
\text { from fatal wound }\end{array}$ & Absent \\
\hline Clothing & $\begin{array}{l}\text { Normally not } \\
\text { disturbed }\end{array}$ & Not disturbed \\
\hline Defense wounds & Often present & Absent \\
\hline $\begin{array}{l}\text { Nature } \\
\text { wounds }\end{array}$ & $\begin{array}{l}\text { Usually chop } \\
\text { wounds, laceration } \\
\text { and stab }\end{array}$ & $\begin{array}{l}\text { Usually } \\
\text { laceration, } \\
\text { abrasion } \\
\text { contusion }\end{array}$ \\
\hline Target Area & No fixed site & Anywhere \\
\hline $\begin{array}{l}\text { Wound } \\
\text { Grouping }\end{array}$ & Irregular & Vulnerable parts \\
\hline Direction & Any Direction & Any Direction \\
\hline Severity & $\begin{array}{l}\text { Mostly severe and } \\
\text { extensive }\end{array}$ & Variable severity \\
\hline $\begin{array}{l}\text { Secondary } \\
\text { Injuries }\end{array}$ & $\begin{array}{l}\text { May be connected } \\
\text { with fight }\end{array}$ & $\begin{array}{l}\text { May be associated } \\
\text { with accident }\end{array}$ \\
\hline $\begin{array}{l}\text { Weapon } \\
\text { available at } \\
\text { crime/accident } \\
\text { scene }\end{array}$ & $\begin{array}{l}\text { Absent, May be } \\
\text { Present }\end{array}$ & $\begin{array}{l}\text { May be Present / } \\
\text { Absent }\end{array}$ \\
\hline
\end{tabular}

\section{IMAGE ACQUISITION AND PRE- PROCESSING}

The images were acquired with color Digital camera under controlled conditions. The camera with a zoom lens $14 \mathrm{x}$ focal length and close-up set 1x. The Images were acquired with $300 \times 400$ pixels in size, for sample of images refer. The controlled condition indicates that there will not be any further damages made to victims' body and at specially wound region. The quality of the image is decisive for identification and classification of wound pattern and weapon, affecting both the ability to detect features under analysis and precision of subsequent measurements. To improve the quality of the images, the images are improved by removing noise and then apply the sharpening so as to get better measurable features. 


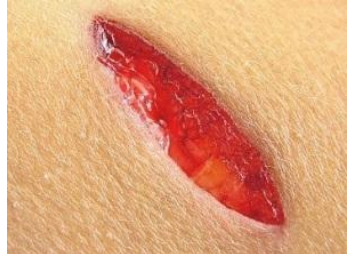

(a)

(b)

Fig 2: Image Samples (a) Stabbed Wound of Double-edge knife (b) Accidental Wound

\subsection{Histogram Equalization}

The Histogram Equalization block enhances the contrast of images by transforming the values in an intensity image so that the histogram of the output image approximately matches a specified histogram. Suppose for an image $f(x, y)$, and its histogram $\mathrm{h}(\mathrm{i})$, the accumulative function of $\mathrm{h}(\mathrm{i})$ as given in Eq.1:

$C(i)=\int_{0}^{i} h(t) d t$

It can be proved that such a transform makes the variable $\mathrm{y}=$ C(i) follow an uniform distribution. Thus, for a 256 gray level image, the histogram equalization can be performed by using the equation Eq.2

$t=\frac{256}{n} C(f(x, y)$

where $\mathrm{n}$ is total number of pixels in the image.

\subsection{Spatial Filtering}

Filtering is fundamental operation in image processing. It can be used for image enhancement, noise reduction, edge detection, and sharpening. Spatial filtering can be performed using the convolution operation as show in Eq. 3

$s(x, y)=\sum_{m=-M / 2}^{M / 2} \sum_{n=-N / 2}^{N / 2} h(m, n) f(s-m, y-n)$ Eq. 3

where $h(m, n)$ is Gaussian filtering mask of size $\mathrm{Mx} \mathrm{N}$.

\section{SEGMENTATION TECHNIQUES}

Segmentation is an important step in the process of recognition of wounds in the image. This step separates the region of interest from the image for further analysis. Digital analysis of images is an exciting research area that requires a synergy between technical, engineering, and biomedical and various other disciplines. Segmentation is an operation that is used to apportioning the images according to similarity, discontinuity or by determining the edges to explore the information. The main objectives of studying of image segmentation techniques is to have a best recognition of wounds of interest and to observe the features in a particular image that can be separated from its background. This is followed by the clustering the pixel corresponding to region, texture, boundary etc. This whole process termed as Segmentation. The various methods that comes under segmentation includes Thresholding based Segmentation, Edge based Segmentation, Region based Segmentation and Clustering Methods. Aim of writing this paper is to arrange the detailed information in this field. Table 3 describes the comparison between segmentation techniques along with their advantages and disadvantages and section 9 concludes the overall study. Image segmentation is to partition an image into significant provinces. Image segmentation, is vital step in image analysis, object recognition, Feature extraction, representation, visualization, and many other image processing tasks.

\subsection{Threshold-Based Segmentation}

Thresholding technique is used to convert a multilevel or a grey scale image into a binary image with the help of histogram of the image. Thresholding refers to an amount or a level. Histogram is the graphical representation of an image having diverse intensities values. To apply Thresholding a preset threshold value $\mathrm{T}$ is used to compare the intensities of the pixels at the peaks, if the pixel is dark it growing darker or white it become whiter. The drawback of Thresholding method is that it needs peaks if there is flat portion it is not able to describe about the object and the background. Determining correct threshold value is another crucial factor.

\subsection{Edge-Based Segmentation}

In this method the images are partitioned by identifying the edges or pixels of rapid transition in intensity. Edge Based approach is done by first detecting the edges or pixels between the different region that have change in intensity and linked those pixels to form closed boundaries. The edges identified by this segmentation technique are often disconnect and to segment the pixel there should have closed connect boundaries. Boundaries are connected by using various morphological operations. These techniques are less immune to noise and if more than two edges have to be determined then it doesn't work well.

\subsection{Region Based Segmentation}

In region-based segmentation technique the similar subset or pixels of an image based upon some criteria are grouped together to form image region. Image regions are constellation of connected pixels with similar belongings. So, region is an image consists of a group of pixels having similar properties. This method is simple and more immune to noise. Edge based technique partition an image into region that is dissimilar set of predefined criteria. This method consists of Region Growing, Region Merging and Region Splitting. Region Growing involves the selection of seed points that means select the entire path where intensity is high and then determine the range of threshold and mark the strongest light point in the respective image. Region Merging refers to merge the entire similar region and Splitting refers to split uncommon region in an image.

\subsection{Clustering}

Clustering Method refers to the pixels having similar properties grouped together known as clusters. Grouping is based on maximizing the similarities, if inter class similarities are increased then the quantity of clusters is automatically increased to get the optimum results. In this it needs to define the centers, one center for each cluster. The center should be far from the others so that the distance could be calculated easily and the data points could predict their clusters very precisely. Then distance between each data point and the clusters centers is calculated. Then data points are assigned to the cluster whose distance from the cluster center is lowest. The distance can be calculated with the help of conventional mathematical perpendicular concept. The distance is calculated and those data points are assigned which are too nearer to cluster. This process is repeated till the shifting is observed in the center of the cluster. The main drawback having this method is to determine the number of clusters in an image.

\section{FEATURES SELECTION AND EXTRACTION}

Many different objects are considered for extensive studies in image-based recognition. This is partly due to the remarkable 
recognition capability of the human visual system, and partly due to numerous important applications for automatic recognition technologies. In addition, technical issues associated with recognition are representative of object recognition in general. In this work, wound and its cause recognition system is proposed and an analysis of the feature selection and extraction is discussed.

\subsection{Feature Selection}

A central issue in the study of wound recognition has been the question of which features of a wound are the most important or informative for recognition. Due to the special geometric shape and appearance of the wounds, instead of using fixed filter that are effective for analyzing stationary signals such as textures. The features extracted using such filters or masks are thought to be more relevant to face recognition, allowing reasonable recognition performance with simple, scalable classifiers. However, with so many proposed features but so little consensus about which feature are better or worse, practitioners lack guidelines to decide which features to use. In the recognition process, there has been enormous effort and emphasis on finding the "optimal" features. This quest may have obscured other important factors that can help clarify the role of feature selection in the overall recognition process. For instance, the performance variation of different features may be due in part to the choice of classifiers or in part to the choice in the range of feature dimension. Furthermore, compressed sensing offers a new perspective on feature selection: it suggests the number of features is much more important than the details of how they are constructed. As long as the number of features is large enough, even randomly chosen features are sufficient to recover the sparse representation. Feature selection in the new context: Thus, it is bound to re-examine feature selection in wound recognition from this new perspective and try to answer the following question: To what extent does the selection of features still matter?

Table 2. List of Different Features Vector

\begin{tabular}{|l|l|}
\hline Features & Details of Features Vector \\
\hline Color & Mean, Variance, Range of RGB \& HSI \\
\hline Texture & GLCM, Correlation \\
\hline Shape & $\begin{array}{l}\text { Tensor Scale, Perimeter, Area, Bounding Box, } \\
\text { Convex Hull, Compactness, Central Moments, } \\
\text { Size Invariant, Eccentricity, Shape Signature } \\
\text { and Shape Matrix }\end{array}$ \\
\hline
\end{tabular}

Feature selection plays a key role in many pattern recognition problems such as image classification. While a great many of features can be utilized to characterize an image, only a few numbers of them are efficient and effective in classification. More features do not always lead to a better classification performance, thus feature selection is usually performed to select a compact and relevant feature subset in order to reduce the dimensionality of feature space, which will eventually improve the classification accuracy and reduce time consumption.

\subsection{Feature Extraction}

In order to obtain an effective feature subset by feature selection, the original feature set must be sufficient. Since low level visual features such as color, texture, and shape are fundamental to characterize images, features of these three types are extracted to compose the pool of features for selection. Color descriptors are extracted from each image by combining different color models and quantization strategies. Texture descriptors characterize the structural pattern of an image. Similar to color and texture descriptor, shape feature is also an important low-level descriptor and has been widely used in image classification and shape descriptors are collected from each image. Note that most of the features have more than one dimension and all feature descriptors consist of multi-dimension feature components.

\section{RECOGNITION METHODOLOGY}

There are many image classification methods, but it remains unclear which methods are most helpful for analyzing and intelligently recognizing wound images. To facilitate this study, some feature extraction algorithms and classifiers are combined to form automatic system with same dataset and then their performance are compared using multiple criteria. This comparative study reveals the general characteristics of the existing methods for automatic recognition of wound images and provides new insights into the strengths and shortcomings of these methods. A pattern class is a family of patterns that share some common properties. Pattern classes are denoted $\omega_{1}, \omega_{2}, \ldots, \omega_{W}$, where $\mathrm{W}$ is the number of classes. Pattern recognition by machine involves techniques for assigning patterns to their classes automatically and with as little human intervention as possible. The work proposes different schemas for recognition that includes the combination of segmentation, feature selection and classifiers. Furthermore, a comparative analysis on these recognition schemas is made.

\subsection{Recognition based on Decision- Theoretic Methods}

Decision-theoretic approached to recognition are based on the use of decision or discriminant functions. Let $x=$ $\left(x_{1}, x_{2}, \ldots, x_{n}\right)^{T}$ represent an $\mathrm{n}$-dimensional pattern vector. For $\mathrm{W}$ pattern classes $\omega_{1}, \omega_{2}, \ldots, \omega_{W}$, the basic problem in decision-theoretic pattern recognition is to find $\mathrm{W}$ decision functions $d_{1}(x), d_{2}(x), \ldots, d_{W}(x)$ with the property that, if a pattern $\mathrm{x}$ belongs to class $\omega_{i}$, then

$d_{1}(x)>d_{2}(x) \quad j=1,2, \ldots, W ; j \neq i$

In other words, an unknown pattern $x$ is said to belong to the $i$ th pattern class if, upon substitution of $\mathrm{x}$ into all decision functions, $d_{i}(x)$ yields the largest numerical value. Ties are resolved arbitrarily. The decision boundary separating class $\omega_{i}$ from $\omega_{j}$ is given by values of $\mathrm{x}$ for which $d_{1}(x)=d_{2}(x)$ or, equivalently, by values of $\mathrm{x}$ for which

$d_{1}(x)-d_{2}(x)=0$

Common practice is to identify the decision boundary between two classes by the single function $d_{i j}(x)=d_{i}(x)-$ $d_{j}(x)=0$. Thus $d_{i j}(x)>0$ for patterns of class $\omega_{i}$ and $d_{i j}(x)<0$ for patterns of class $\omega_{j}$. The principal objective is to develop an approach for finding decision function that satisfy Eq. 4

\subsection{Recognition based on Structural Methods}

The techniques discussed in section 7.1 deal with patterns quantitatively and largely ignore any structural relationships inherent in a pattern's shape. The structural methods, however, seek to achieve pattern recognition by capitalizing precisely on these types of relationships. In this method, two basic approaches for the recognition of boundary shapes based on string representations is introduced. Strings are the most practical approach in structural pattern recognition. "Matching 
Shape Numbers" procedure analogous to the minimum distance concept for pattern vectors can be formulated for the comparison of region boundaries that are described in terms of shape numbers. The degree of similarity, k, between two region boundaries (shapes) is defined as the largest order for which their shape numbers still coincide. Let $a$ and $b$ denote shape numbers of closed boundaries represented by 4directional chain codes. These two shapes have a degree of similarity $\mathrm{k}$ if

$$
\begin{array}{ll}
s_{j}(a)=s_{j}(b) & \text { for } \mathrm{j}=4,6,8, \ldots, \mathrm{k} \\
s_{j}(a)=s_{j}(b) & \text { for } j=k+2, k+4, \ldots
\end{array}
$$

Where $s$ indicates shape number and the subscript indicates order. The distance between two shapes $a$ and $b$ is defined as the inverse of their degree of similarity:

$D(a, b)=\frac{1}{k}$

This distance satisfies the following properties: $D(a, b) \geq 0$, $D(a, b)=0$ iff $\mathrm{a}=\mathrm{b}$ and $D(a, c) \leq \max [D(a, b), D(b, c)]$. Either $\mathrm{k}$ or D may be used to compare two shapes. If the degree of similarity is used, the larger $\mathrm{k}$ is, the more similar the shapes are ( $\mathrm{k}$ is infinite for identical shapes). The reverse is true when the distance measure is used. Another approach is string matching, suppose that two region boundaries a and $\mathrm{b}$, are coded into strings denoted $a_{1}, a_{2}, \ldots, a_{n}$ and $b_{1}, b_{2}, \ldots, b_{m}$ respectively. Let $\alpha$ represent the number of matches between the two strings, where a match occurs in the $k$ th position if $a_{k}=b_{k}$. The number of symbols that do not match is

$\beta=\max (|a|,|b|)-\alpha$

Where $|\arg |$ is the length (number of symbols) in the string representation of the argument. It can be shown that $\beta=0$ if and only if $a$ and $b$ are identical. A simple measure of similarity between $\mathrm{a}$ and $\mathrm{b}$ is the ratio

$R=\frac{\alpha}{\beta}=\frac{\alpha}{\max (|a|,|b|)-\alpha}$

Hence $\mathrm{R}$ is infinite for a perfect match and 0 when none of the corresponding symbols in a and $\mathrm{b}$ match i.e., $\alpha=0$ in this case. Because matching is done symbol by symbol, the starting point on each boundary is important in terms of reducing the amount of computation.

\section{COMPARATIVE ANALYSIS AND DISCUSSION}

The methods with combination of shape features vector has achieved an average accuracy more than $85 \%$ and can be adopted in specific situations to aid investigators or experts in preliminarily wound recognition. Furthermore, some methods requiring fewer computational resources and less time could be applied in remote places or mobile devices to assist individuals in understanding the condition of the body. In addition, it would be helpful to accelerate the development of innovative approaches and to apply these methods to assist investigators. For the comparison of accuracy result of various schemas proposed in this work, refer figure 3 .

\subsection{Comparative Analysis of Segmentation Techniques}

Four segmentation techniques are considered for the comparative analysis work: threshold-based, edge-based, region-based and clustering techniques. As the main object considered in this recognition is wounds that are created by the weapons stabbed or accidental, as per the characteristics of weapons, wounds structure appears on the human body. So, it is required to concentrate on the shape of wounds rather than other features such as color and textures. The number of wounds available on human body are usually single or multiple. All the four segmentation techniques are applied on the same set of images and as a result, the threshold-based segmentation has given better result compared to the other

\begin{tabular}{|c|c|c|c|}
\hline Segmentation & Description & Advantages & Disadvantages \\
\hline $\begin{array}{l}\text { Threshold- } \\
\text { based } \\
\text { Segmentation }\end{array}$ & $\begin{array}{l}\text { Subject to } \\
\text { the } \\
\text { histogram of } \\
\text { an image }\end{array}$ & $\begin{array}{l}\text { Simple } \\
\text { approach } \\
\text { that doesn't } \\
\text { require prior } \\
\text { knowledge } \\
\text { of image }\end{array}$ & $\begin{array}{l}\text { Doesn't work } \\
\text { proper if edges } \\
\text { are more in } \\
\text { images }\end{array}$ \\
\hline $\begin{array}{l}\text { Edge-based } \\
\text { Segmentation }\end{array}$ & $\begin{array}{l}\text { Subject to } \\
\text { the pixels } \\
\text { with } \\
\text { different } \\
\text { intensities }\end{array}$ & $\begin{array}{l}\text { Detection of } \\
\text { the edges is } \\
\text { easy in } \\
\text { images }\end{array}$ & $\begin{array}{l}\text { Less resist to } \\
\text { noise and } \\
\text { doesn't work if } \\
\text { the edges are } \\
\text { not clearly } \\
\text { defined. }\end{array}$ \\
\hline $\begin{array}{l}\text { Region-based } \\
\text { Segmentation }\end{array}$ & $\begin{array}{l}\text { Group of } \\
\text { pixels with } \\
\text { similar } \\
\text { parameters } \\
\text { that } \\
\text { constitute } \\
\text { the region }\end{array}$ & $\begin{array}{l}\text { Works well } \\
\text { when region } \\
\text { homogeneity } \\
\text { standards are } \\
\text { defined } \\
\text { clearly and } \\
\text { more resist } \\
\text { to noise. }\end{array}$ & $\begin{array}{l}\text { Two stage } \\
\text { segmentation } \\
\text { method that } \\
\text { increases cost } \\
\text { of } \\
\text { implementation }\end{array}$ \\
\hline $\begin{array}{l}\text { Clustering- } \\
\text { based } \\
\text { Segmentation }\end{array}$ & $\begin{array}{l}\text { Group of the } \\
\text { pixels with } \\
\text { similar } \\
\text { parameters } \\
\text { that defines } \\
\text { cluster } \\
\text { values with } \\
\text { visible } \\
\text { intensities }\end{array}$ & $\begin{array}{l}\text { Easy } \\
\text { Detection } \\
\text { and } \\
\text { implementati } \\
\text { on }\end{array}$ & $\begin{array}{l}\text { Defining the } \\
\text { value of cluster } \\
\text { is required }\end{array}$ \\
\hline
\end{tabular}
three techniques.

Table 3. Comparison of Segmentation Techniques

\subsection{Comparative Analysis of Feature Vectors}

Selecting features is an important factor in the recognition process. As wound patterns are involved, features selected are shapes parameters which plays major role in detecting the pattern and few color features such as RGB and HSI mean, variance and range and texture features such as GLCM and Correlation are considered to support and improve the overall detection accuracy of the proposed problem statement.

\subsection{Comparative Analysis of Recognition Methodologies}

It has been felt that in the past there was an unbalanced development between theory and practice in pattern recognition. Many theoretical results, especially in connection with the decision-theoretic approach, have been published. Practical applications have been gradually emphasized during the last five years, particularly in medical and remote sensing areas. Most of the practical results are considered inconclusive and require further refinement. Implementation of a practical system is often on a general-purpose computer 


\section{Comparative Analysis of Methodologies}

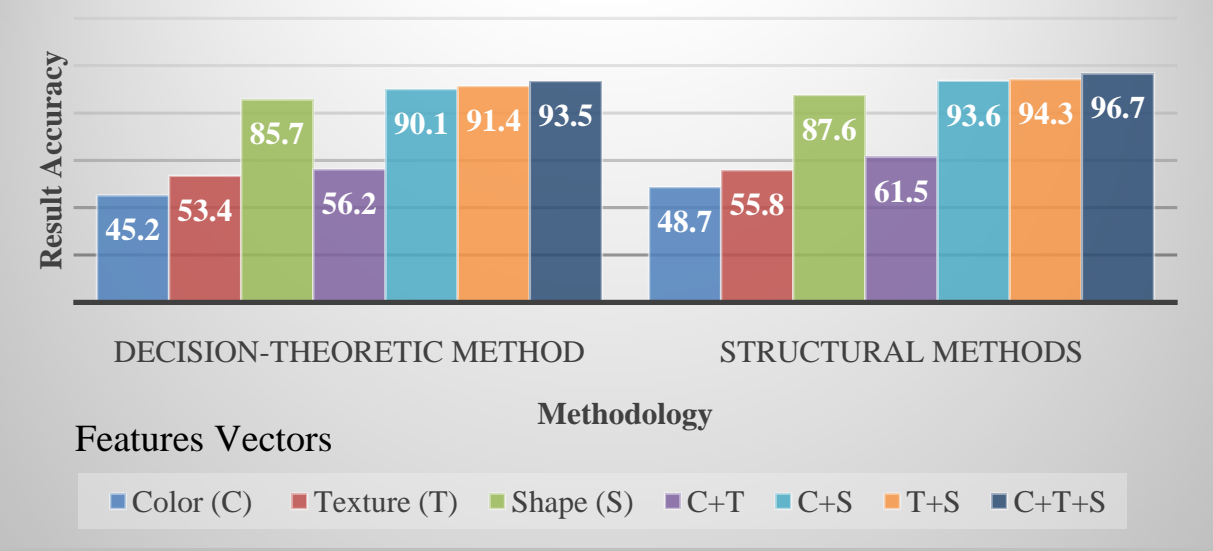

Fig 3: Graph showing the accuracy results of the proposed schemas

In the decision-theoretic approach, still there is need for effective and efficient feature extraction and selection techniques, particularly in nonparametric and small sample situations. The computational complexity of pattern recognition systems, in terms of time and memory, should be an interesting subject for investigation. In the structural approach, the problem of primitive extraction and selection certainly needs further attention. An appropriate selection of the pattern grammar directly affects the computational complexity or analysis efficiency of the resulting recognition system. Grammatical inference algorithms which are computationally feasible are still highly in demand.

\section{CONCLUSION}

In this work, briefly explained the various segmentation techniques with their output results including advantages and disadvantages. The image segmentation methods that mentioned in this paper are used in many technologies including pattern recognition as well as for the image analysis. The result shows that the threshold-based segmentation technique is better for the recognition of stabbed and accidental wounds. This has been contended both theoretically and experimentally that exploiting features is critical for highperformance wound recognition. With properly harnessed, the choice of features becomes more important than the number of features used. As in this work, the wound patterns and weapons are involved, the shape features will be important feature vector where as some few color and texture features will play minor role in the recognition i.e., supporting features for the shape features to improve the overall accuracy of the detection. It is believed that the proposed recognition framework provides new solutions for such problem statements. Pattern recognition is generally categorized according to the type of learning procedure used to generate the output value. In simple sense pattern recognition is the heart of all scientific inquiry, including understanding ourselves and the real-world around us. Now a day the development of pattern recognition is increasing very fast. In this paper, navigated pattern recognition methods, in addition, two approaches are used for comparative analysis, out of that structural method has given better result than the other decision-theoretic method. It is an important trend to use pattern recognition on engineering applications; one should make efforts on pattern recognition.

\section{REFERENCES}

[1] Javaregowda Vinay et.al, (2017), “A Study on Postmortem Wound Dating by Grossand Histopathological Examination of Abrasions", Am J Forensic Med Pathol Vol 38, pp: 167-173

[2] Naveen Tokas et al, (2016) "Comparison of Digital Image Segmentation Techniques- A Research Review", International Journal of Computer Science and Mobile Computing, Vol.5 Issue.5, pp. 215-220

[3] Xuan Zhou, Jiajun Wang (2015), "Feature Selection for Image Classification Based on a New Ranking Criterion", Journal of Computer and Communications, Vol 3, pp: 74-79

[4] Dayanand G Savakar, Anil Kannur (2015) "A Genetic algorithm and Bayesian approach for identification \& classification of weapon based on the stab wound patterns caused by different sharp metal", International Journal of Computer Engineering and Applications, Volume IX, Issue I, pp: 01-12.

[5] Dayanand G. Savakar and Anand Ghuli, (2015), "Digital Watermarking as distributed noise by DWT, Fast Fourier Transformation and Fast Walsh-Hadamard Transform to study the sensitivity between Robustness and Fidelity", International Journal of Computer Application, Issue 5, Vol.1, pp 102-107.

[6] Dayanand G. Savakar and Anand Ghuli, (2014), "Digital Watermarking-A Combined Approach by DWT, Chirp-Z and Fast Walsh-Hadamard Transform", International Journal of Computer Technology and Applications (IJCTA), Vol. 5 No.6, pp 2006-2010, ISSN 2229-6093.

[7] Song Bo, (2012) "Automated wound identification system based on image segmentation and Artificial Neural Networks", IEEE International Conference on Bioinformatics and Biomedicine, pp: 11-16.

[8] B.S.Anami, Sunanda Biradar and D.G.Savakar, (2013), "Identification and Classification of Similar looking food grains", International Conference on Communication and Electronics System Design, ICCESD.

[9] Gitto L., Vullo A., Demari G.M., (2012) "Identification of the murder weapon by the analysis of a typical pattern of sharp force injury", Italian Journal of Legal Medicine, Vol: 01, Issue No. 1, pp: 04-14.

[10] Ying Bai; Dali Wang, (2011)"Evaluate and identify optimal weapon systems using fuzzy multiple criteria 
decision making", Proceedings of IEEE International Conference on Fuzzy Systems (FUZZ), pp: 1510-1515.

[11] Suapang P., et.al..,(2011), "Tool and Firearm Identification System Based on Image Processing", Proceedings of 11th International Conference on Control, Automation and Systems (ICCAS), pp: $178-182$

[12] Kaliszan M., Karnecki K., Akçan R., (2011) "Striated abrasions from a knife with non-serrated bladeidentification of the instrument of crime on the basis of an experiment with material evidence", International Journal of Legal Medicine, Vol: 125, Issue No. 5, pp: 745-748

[13] Ajay Kumar N, et.al., (2011) "Automated human identification using ear imaging", Journal of Pattern Identification, pp: 1-13.

[14] Basavaraj S. Anami and Dayanand G. Savakar, (2011), "Suitability of Feature Extraction Methods in Recognition and Classification of Grains, Fruits and Flowers", International Journal of Food Engineering, Volume 7, Issue 1, Article 9, pp: 1-28, Publisher: Berkeley Electronic Press, Berkeley, U.S.A.

[15] Francisco Veredas, et.al., (2010)"Binary Tissue Classification on Wound Images With Neural Networks and Bayesian Classifiers", IEEE transactions on medical imaging, Vol: 29, Issue 2, pp: 410-426.

[16] B. S. Anami, Dayanand G. Savakar, (2009), "Effect of Foreign Bodies on Identification and Classification of Bulk Food Grains Images", Journal of Applied Computer Science and Mathematics, Vol 3(6), pp:77- 83.

[17] F.A. Andaló, A.V. Miranda, A.X.Falcão, (2009), "Shape feature extraction and description based on tensor scale", Journal of Pattern Recognition, Elsevier Ltd, pp:1-11.
[18] Jamieson A., Monessen A., (2009) "Wiley Encyclopedia of Forensic Sciences", Wiley \& Sons Ltd Edition, UK, pp: 2647-2649.

[19] M. Brandon Westover and Joseph A. O'Sullivan, (2008) "Achievable Rates for Pattern Identification", IEEE transactions on Information Theory, Vol: 54, Issue No. 1, pp: 299-320.

[20] Li Dongguang, (2008) "Firearm Identification System Based on Ballistics Image Processing", Proceedings of CISP '08, Congress on Image and Signal Processing Vol: 3, pp: $149-154$

[21] Jie Liu1, Jigui Sun, Shengsheng Wang, (2006) "Pattern Identification: An overview", IJCSNS International Journal of Computer Science and Network Security, Vol:6, Issue No.6, pp: 57-61

[22] Qi Peter Li, and Biing-Hwang Juang, (2006) "Study of a Fast-Discriminative Training Algorithm for Pattern Identification", IEEE transactions on neural networks, Vol: 17, Issue No. 5, pp-1212-1221

[23] T. Plattner, B. Kneubuehl, M. Thali, U. Zollinger, (2003) "Gunshot residue patterns on skin in angled-contact and near contact gunshot wounds", Forensic Science International, Elsevier publication, Vol. 138, pp:68-74.

[24] Candida Ferreira, (2001) "Gene Expression Programming: A New Adaptive Algorithm for Solving Problems", Journal of Complex Systems, Vol. 13, issue 2, pp: 87-129.

[25] Hugar B.S. et al., (2012) "Study of defense injuries in homicidal deaths - An autopsy study", Journal of Legal Medicine, Vol: 19, Issue No. 4, pp: 207-215. 